\title{
Primary Presentation of Ovarian Cancer with Bladder Outlet Obstruction/Chronic Urinary Retention in a 12-Year Old Female
}

\author{
Charles Azuwike Odoemene ${ }^{1 *}$, Ijeoma Ezeome ${ }^{2}$, Okechukwu Charles Okafor ${ }^{3}$ \\ ${ }^{1}$ Alex Ekwueme Federal University Teaching Hospital, Abakaliki, Nigeria \\ ${ }^{2}$ University of Nigeria Teaching Hospital, Enugu, Nigeria \\ ${ }^{3}$ Department of Morbid Anatomy, University of Nigeria Teaching Hospital, Enugu, Nigeria \\ Email: *odoemenec@yahoo.com, ezeomeij@gmail.com, okechukwu.okafor@gmail.com
}

How to cite this paper: Odoemene, C.A., Ezeome, I. and Okafor, O.C. (2021) Primary Presentation of Ovarian Cancer with Bladder Outlet Obstruction/Chronic Urinary Retention in a 12 -Year Old Female. Open Journal of Urology, 11, 233-239. https://doi.org/10.4236/oju.2021.117021

Received: April 29, 2021

Accepted: July 6, 2021

Published: July 9, 2021

Copyright $\odot 2021$ by author(s) and Scientific Research Publishing Inc. This work is licensed under the Creative Commons Attribution International License (CC BY 4.0).

http://creativecommons.org/licenses/by/4.0/

\begin{abstract}
Urinary retention in women is rare and is more frequently described as case reports or small case series. The female/male ratio is 1:13 with about 3 cases per 100,000 women every year We report a case of a 12-year old female student. She presented with progressive weight loss, worsening lower urinary tract symptoms with distended lower abdomen of 10 weeks duration. Physical examination revealed a mobile tender firm pelvic mass, 18 centimeters $(\mathrm{cm}) \times$ $16 \mathrm{~cm}$ in size. Laboratory and imaging studies showed obstructive nephropathy and uropathy respectively. She was worked up and had uneventful exploratory laparotomy with right salpingo-oophorectomy, urinary bladder diverticulectomy and pelvic lymphadenectomy. Histopathology of the pelvic mass showed ovarian dysgerminoma with lymph node metastasis. She responded very well to chemotherapy and resumed her school activities. Bladder outlet obstruction is relatively rare in females and in the index patient, ovarian dysgerminoma is the cause leading to obstructive nephropathy and uropathy.
\end{abstract}

\section{Keywords}

Bladder Outlet Obstruction, Chronic Urinary Retention, Pelvic Mass, Ovarian Dysgerminoma, Chemotherapy

\section{Introduction}

Urinary retention implies a difficulty in passing urine or completely emptying the urinary bladder and it can be acute or chronic. Urinary retention in women is rare with about 3 cases per 100,000 women every year [1]. The female/male ratio is $1: 13$ [1] [2]. In short, urinary retention in females is more frequently de- 
scribed as case reports or small case series with unusual causes [2]. Two common causes of chronic urinary retention in women are detrusor dysfunction and obstruction [1]. Urinary symptoms such as hesitancy, straining, poor urinary stream and feeling of incomplete emptying of the urinary bladder are clearly suggestive of bladder outlet obstruction in women [3] [4] [5].

Anatomical non-iatrogenic causes of bladder outlet obstruction in females include pelvic organ prolapse, vaginal masses like cysts, urethral pathology like diverticulum, carcinoma, gynaecological lesions like large ovarian cysts, tumors, cervical, uterine tumors and pregnancy due to uterine displacement [4].

We present the case of a 12-year old female student with ovarian cancer who presented with lower urinary tract symptoms and chronic urinary retention with urinary tract infection (UTI) and overflow incontinence to the urologist and her successful management.

\section{Case Report}

Miss A, a 12-year old Nigerian student presented at the accident and emergency department with about 3 months worsening symptoms of progressive weight loss, daytime frequency, nocturia, overflow incontinence, feeling of incomplete emptying of the bladder and lower abdominal pain. There was no haematuria.

She was cachectic, clinically pale, anicteric. The chest was unremarkable. There was lower abdominal distension. A tender mobile mass $18 \mathrm{~cm} \times 16 \mathrm{~cm}$ which was firm in consistency was palpated. A working diagnosis of bladder outlet obstruction secondary to a pelvic mass was made. She was aseptically catheterized with a size 12F (all silicone) two-way Foley catheter and 350 mililiters (mLs) of turbid offensive urine drained.

Haematological investigations, serum electrolytes, urea and creatinine, etc. are as shown in Table 1.

Abdominopelvic ultrasonography showed a solid pelvic mass posterior to and compressing the urinary bladder with hypoechoic and highly echogenic areas measuring $18.94 \times 11.24 \mathrm{~cm}$, there was also bilateral hydronephrosis with hydroureter.

Intravenous urography after normalization of serum electrolyte, urea and creatinine showed bilateral hydronephrosis and hydroureter, bladder diverticulum in keeping with bladder outlet obstruction (Figure 1).

The anemia was corrected by blood transfusion, the UTI treated with ceftriaxone. The patient was worked up and prepared for laparotomy. Via a mid-line abdominal incision, the peritoneum and pelvis were explored. The mass was mobilized off the posterior wall of the urinary bladder. Within the mass were embedded the right ovary and the right fallopian tube. There was pelvic and iliac lymphadenopathy. A urinary bladder diverticulum was noted. The mass with the right ovary and right fallopian tube within was resected. Pelvic and iliac lymphadenectomy was done. She had an uneventful post-operative recovery. The indwelling urethral catheter was removed on the seventh-day post-operatively. The 
Table 1. Haematological and other laboratory investigations.

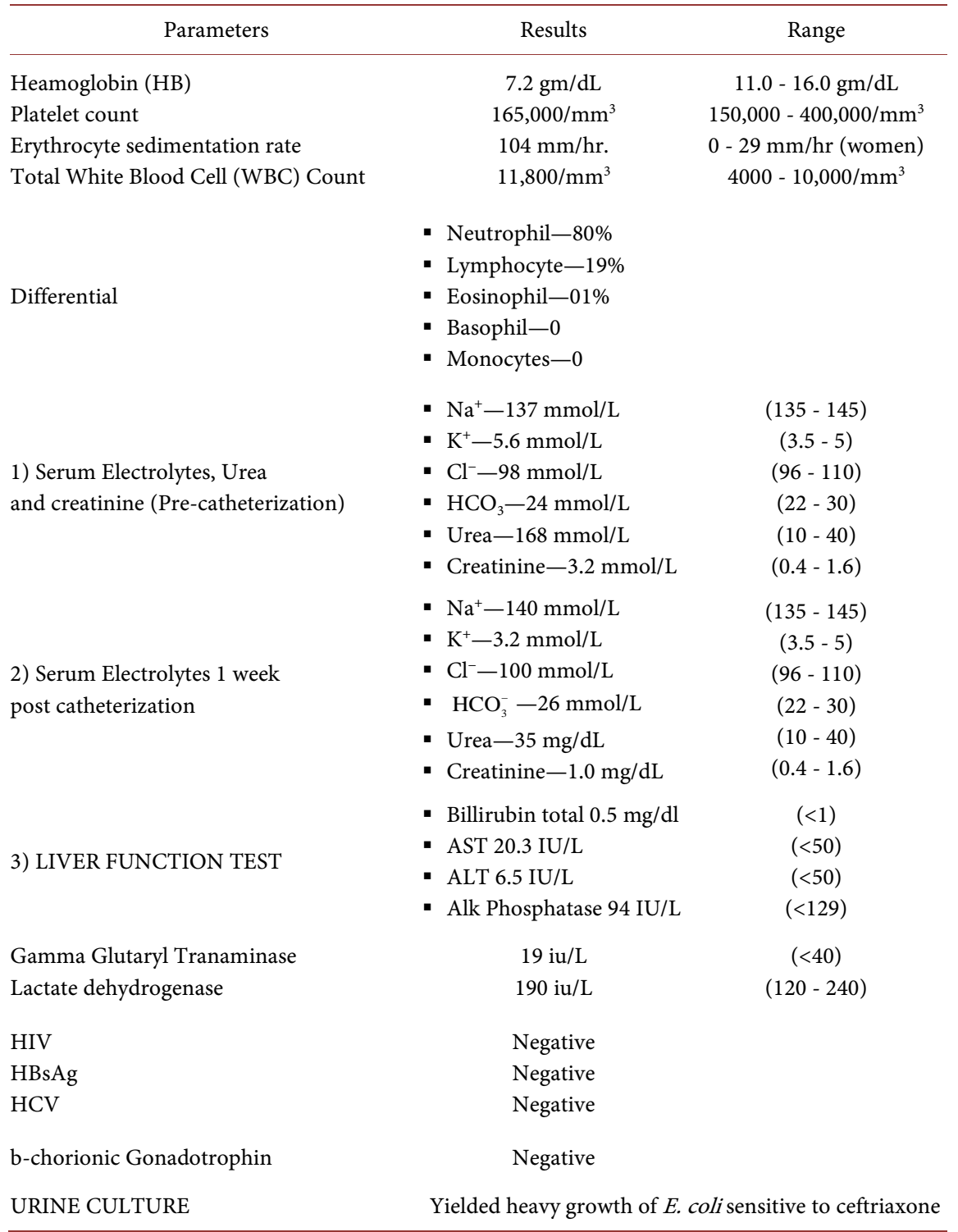

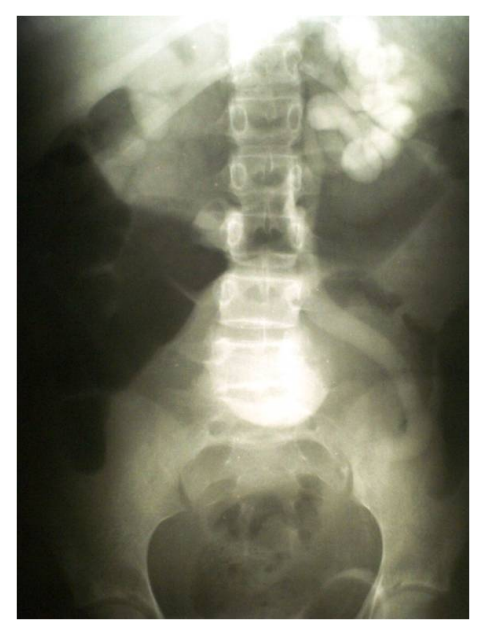

Figure 1. Intravenous urography showing hydronephrosis with hydroureter. 
patient was discharged on the tenth-day post-operatively to the outpatient department. Histology report showed ovarian dysgerminoma. The sections from the ovary and retroperitoneal lymph nodes are similar and show a malignant germ cell tumour made up of broad and thin trabeculae of pleomorphic malignant cells. These cells have abundant amphophilic cytoplasm and large vesicular nuclei with a prominent eosinophilic nucleolus. Between the tumour trabeculae, there are fibrous connective tissue columns that contain many lymphocytes. Within the tumour masses, there are multinucleated giant tumour cells of trophoblastic type. Some of the blood vessels within the tumour show intravascular tumour dissemination (Figure 2, Figure 3).

Patient was referred to the oncologist for chemotherapy and she did well after the course of chemotherapy and resumed her academic activities. She was lost to follow up after 10 months.

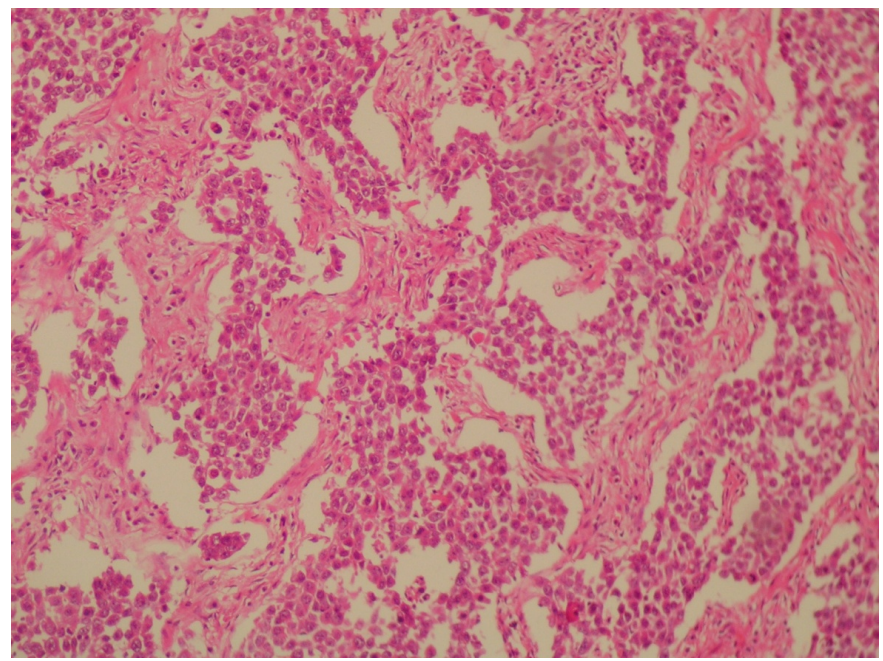

Figure 2. H\&E $\times 150$ magnification ovarian dysgerminoma. There are anastomosing trabeculas of tumor cells separated by fibrous bands that contain lymphocytes.

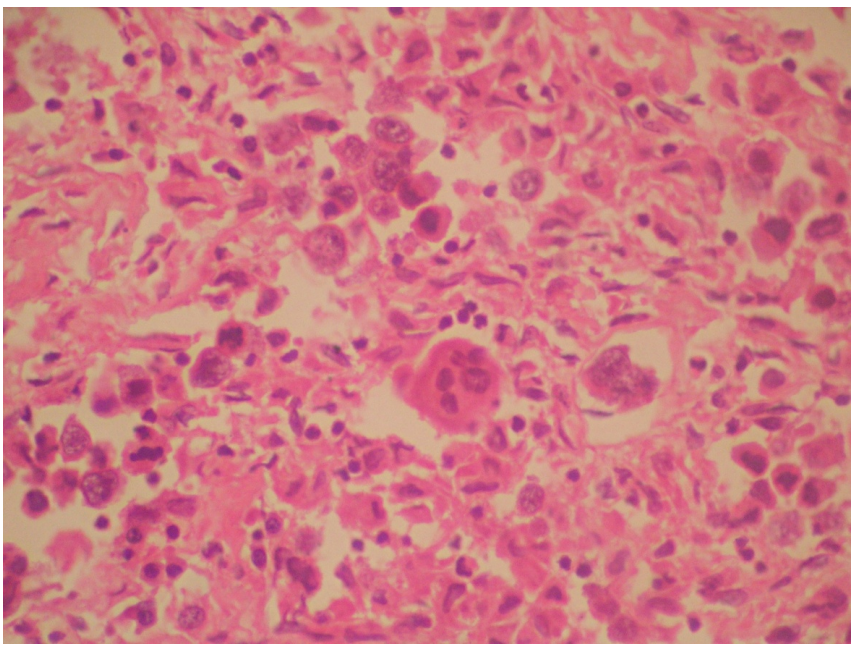

Figure 3. H\&E $\times 300$ magnification ovarian dysgerminoma. Note the frequent multinucleated tumor giant cells among the other tumor cells. 


\section{Discussion}

Although relatively common in men, bladder outlet obstruction is relatively uncommon in women in clinical practice and the etiological factors more diverse in females than in males [3]. The mechanism of obstruction could be urethral compression, bladder neck distortion or luminal occlusion [2]. In the index patient, the ovarian tumor compressed and distorted the bladder neck causing lower urinary tract symptoms, impaired detrusor emptying with increasing residual urine volume. Furthermore, there was associated daytime urinary frequency, nocturia, urgency, poor urinary stream, intermittency, terminal dribbling and feeling of incomplete emptying of the urinary bladder. Storage and voiding symptoms can coexist in bladder outlet obstruction in females making it a challenge in clinical practice to make an accurate diagnosis and offering the best form of treatment [6]. Both urgency urinary incontinence and overflow incontinence coexisted in this patient. Urinary incontinence adversely impacts the patient, family members and the health care system with abstinence from routine activities, increased rate of depression and reduced quality of life [7]. The index patient experienced all these and dropped out of school with depression. In addition, the patient with overflow urinary incontinence has high residual urine volume with associated high pressure within the bladder which at this stage is considered as high-pressure chronic retention and can cause renal impairment [7]. The index patient at presentation, laboratory and ultrasound studies showed obstructive nephropathy and uropathy respectively. A total of $350 \mathrm{~mL}$ turbid offensive urine was drained from the urinary bladder on aseptic urethral catheterization and the patient was admitted and monitored for complications. The patient in the first 72 hours following catheter insertion made a daily average of 3 liters of urine which was promptly replaced with intravenous fluids to avoid dehydration and shock. Drainage of more than $300 \mathrm{~mL}$ of urine from the bladder after voiding suggests urinary retention [1], and the condition is best managed in a hospital setting with the patient monitored for post obstructive diuresis [7]. Imaging studies like abdominopelvic ultrasound, CT imaging have been advocated in the investigation of these patients [1] [3] [5] [6] [8] [9]. Abdominopelvic ultrasonography was utilized to unravel the etiology of this condition. Furthermore, ultrasonography could be useful in estimating the residual urine volume [3] [5] and detection of greater than $200 \mathrm{~mL}$ of urine in the bladder after voiding is suggestive of overflow urinary incontinence [10]. This patient did not experience acute urinary retention. Acute urinary retention in females is due to impacted pelvic masses that displace the cervix superiorly and anteriorly compressing the lower bladder leading to obstruction of the internal urethral orifice [11]. The pelvic mass was mobile in the index patient and not impacted with the internal urethral orifice partially obstructed. The lower abdominal pain the patient had was due to pressure from the mass and urinary tract infection caused by E. coli.

While lactate dehydrogenase (LDH) levels are known to be elevated in some patients with dysgerminoma, our patient had normal values of LDH and $\beta$-cho- 
rionic gonadotrophin $(\beta \mathrm{HCG})$. She had complete resection of the mass with right salpingo-oophorectomy followed by chemotherapy with bleomycin, etoposide and cisplatin (BEP). Even patients with incompletely resected dysgerminoma can be rendered disease-free with a combination of cisplatin, vinblastin, and bleomycin (PVB) [12]. A number of patients had one or more successful pregnancies following unilateral salpingo-oophorectomy [13]. However, our patient was lost to follow up after 10 months of having resumed her academic activities and in excellent health.

\section{Conclusion}

Although relatively common in men with voiding dysfunction, bladder outlet obstruction is relatively rare in women. In this patient right ovarian cancer was the cause of complications of obstructive uropathy and nephropathy, the patient and her relative concern was the disabling lower urinary tract symptoms oblivious of the underlying pathology. The onus thus lies on the attending clinician to evaluate the patient diligently and unravel the pathology and in this case, a right ovarian dysgerminoma which responded excellently to chemotherapy with the patient resuming her academic activities.

\section{Author Contributions}

Study design: Dr. Charles A. Odoemene, Dr. Mrs. Ijeoma Ezeome, Dr. Okechukwu Charles Okafor.

Data acquisition: Charles A. Odoemene, Dr. Mrs. Ijeoma Ezeome, Dr. Okechukwu Charles Okafor.

Data analysis: Charles A. Odoemene, Dr. Mrs. Ijeoma Ezeome.

Drafting of the manuscript: Charles A. Odoemene, Dr. Mrs. Ijeoma Ezeome, Dr. Okechukwu Charles Okafor.

Critical revision of the manuscript: Charles A. Odoemene, Dr. Mrs. Ijeoma Ezeome, Dr. Okechukwu Charles Okafor.

\section{Parents Informed Consent}

We attest that the patient's parents gave consent for the publication of this case.

\section{Conflicts of Interest}

The authors have no conflicts of interest.

\section{References}

[1] Dougherty, J.M. and Rawla, P. (2021) Female Urinary Retention [Updated 2021 Mar. 29]. Stat Pearls [Internet] Treasure Island (FL), Stat Pearls Publishing. https://www.ncbi.nlm.nih.gov/books/NBK538497/

[2] Mevcha, A. and Drake, M.J. (2010) Etiology and Management of Urinary Retention in Women. Indian Journal of Urology, 26, 230-235. https://doi.org/10.4103/0970-1591.65396 
[3] Yande, S. and Joshi, M. (2011) Bladder Outlet Obstruction in Women. Journal of Mid-Life Health, 2, 11-17. https://doi.org/10.4103/0976-7800.83257

[4] Dmochowski, R.R. (2005) Bladder Outlet Obstruction: Etiology and Evaluation. Reviews in Urology, 7, S3-S13.

[5] Shaih, N.A., Hindu, K.A., Shaikh, G.S. and Soornro, A.A. (2015) Bladder Outlet Obstruction (BOO) in Female: Etiology and Management. Rawal Medical Journal, 40, 289-291.

[6] Raheem, A.A. and Madersbacher, H. (2013) Voiding Dysfunction in Women; How to Manage It Correctly. Arab Journal of Urology, 11, 319-330. https://doi.org/10.1016/j.aju.2013.07.005

[7] Mangir, N. and Chapple, C. (2020) Management of Urinary Incontinence in Men. Trends in Urology \& men's Health, 11, 18-22. https://doi.org/10.1002/tre.740

[8] Selcuk, I., Boyraz, G. and Tuncer, Z.S. (2013) Acute Urinary Retention Due to a Giant Ovarian Tumour in Reproductive Ages: Case Report. Turkiye Klinikleri Jinekoloji Obstetrik, 23, 207-210.

[9] Basson, J., Van der Walt, C.I.E. and Heyns, C.F. (2013) Urinary Retention in Women. CME, 31, 182-184.

[10] Tran, L.N. and Puckett, Y. (2021) Urinary Incontinence [Updated 2021 Apr. 13]. Stat Pearls [Internet] Treasure Island (FL), Stat Pearls Publishing.

https://www.ncbi.nlm.nih.gov/books/NBK559095/

[11] Yang, J.M. and Huang, W.C. (2002) Sonographic Findings of Acute Urinary Retention Secondary to an Impacted Pelvic Mass. Journal of Ultrasound in Medicine, 21, 1165-1169. https://doi.org/10.7863/jum.2002.21.10.1165

[12] Williams, S.D., Blessing, J.A., Hatch, K.D. and Homesley, H.D. (1991) Chemotherapy of Advanced Dysgerminoma: Trails of the Gynecologic Oncology Group. Journal of Clinical Oncology, 9, 1950-1955. https://doi.org/10.1200/JCO.1991.9.11.1950

[13] Thomas, G.M., Dembo, A.J., Hacker, N.F. and Depetrillo, A.D. (1987) Current Therapy for Dysgerminoma of the Ovary. Obstetrics \& Gynecology, 70, 268-275. 